\title{
Implementation Plan for the Office of Nuclear Energy Knowledge Management Program
}

December 2011

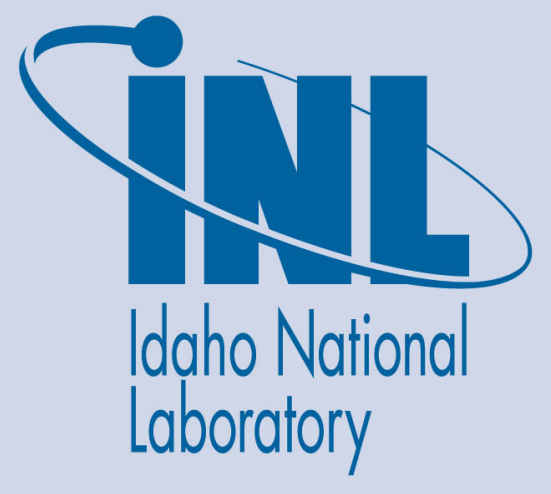

The INL is a U.S. Department of Energy National Laboratory operated by Battelle Energy Alliance 


\title{
Implementation Plan for the Office of Nuclear Energy Knowledge Management Program
}

December 2011

\author{
Idaho National Laboratory \\ Idaho Falls, Idaho 83415
}

http://www.inl.gov

Prepared for the

U.S. Department of Energy

Office of Nuclear Energy

Under DOE Idaho Operations Office

Contract DE-AC07-05ID14517 


\section{Table of Contents}

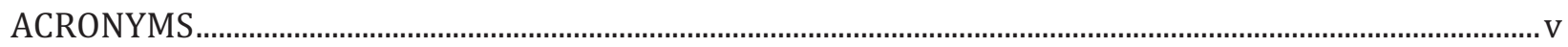

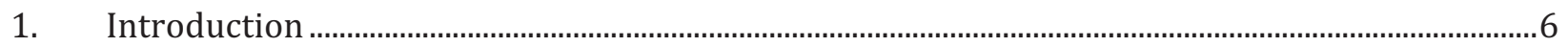

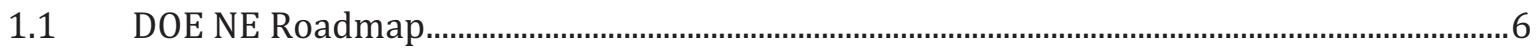

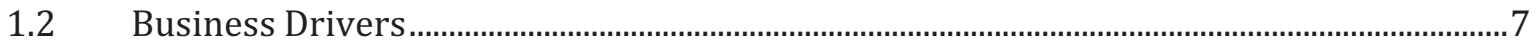

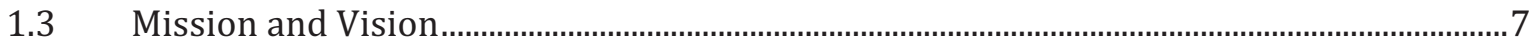

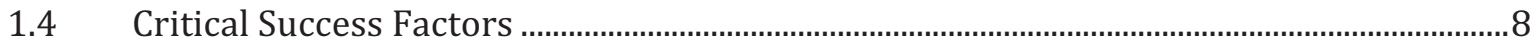

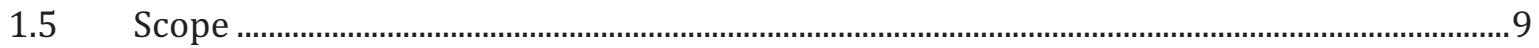

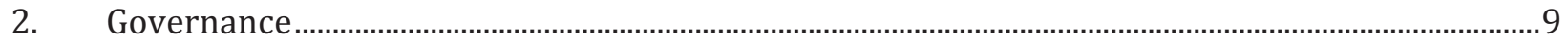

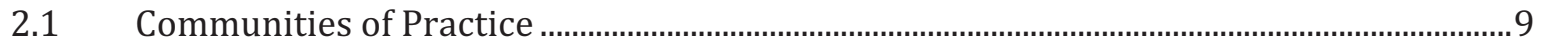

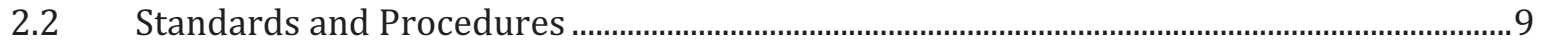

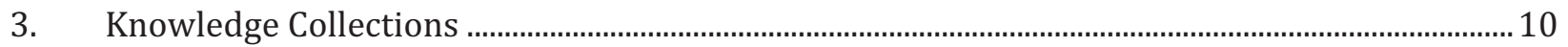

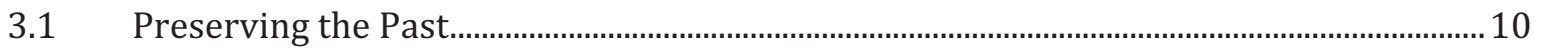

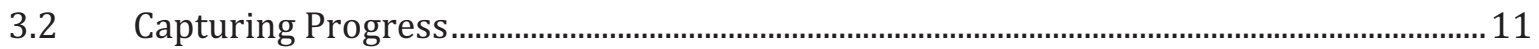

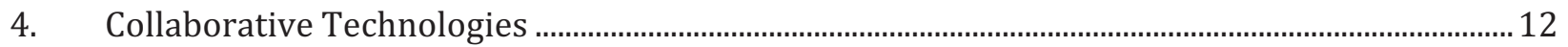

$4.1 \quad$ Architecture

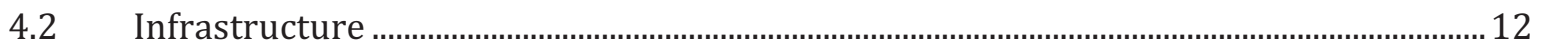

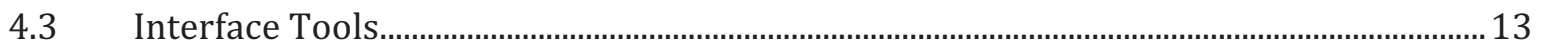

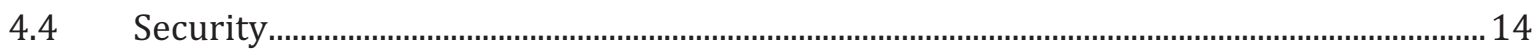

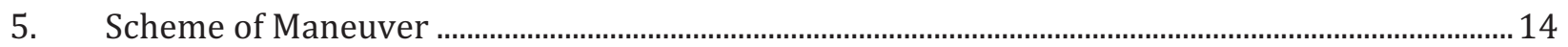

$5.1 \quad$ Collaboration Portal - Extranet .................................................................................................... 14

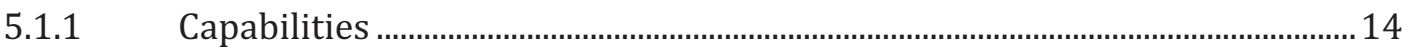

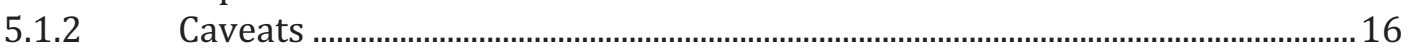

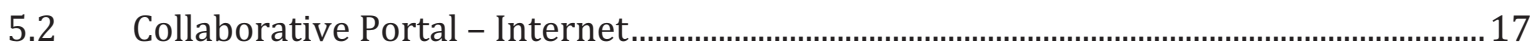

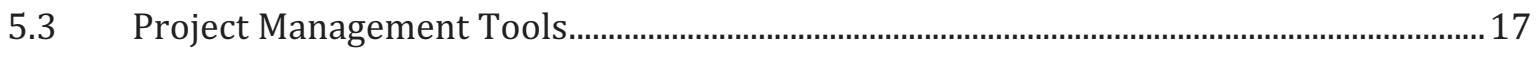

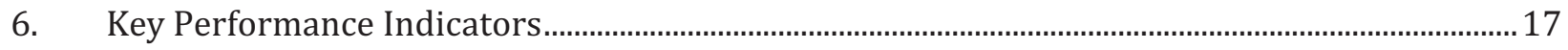

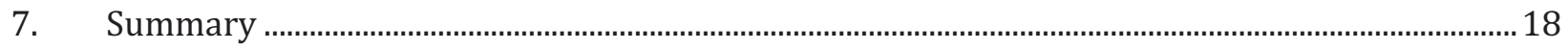




\section{ACRONYMS}

ARC Advanced Reactor Concepts

AT Applied Technology

DOE Department of Energy

DOE-NE Department of Energy, Office of Nuclear Energy

FFTF Fast Flux Test Facility

INL Idaho National Laboratory

KM Knowledge Management

LWRI Light Water Reactor Improvements

LWRS Light Water Reactor Sustainability

NE nuclear energy

NE KM Office of Nuclear Energy, Knowledge Management Program

NGNP Next Generation Nuclear Plant

RW Office of Civilian Radioactive Waste Management 


\section{Implementation Plan for the Office of Nuclear Energy Knowledge Management Program}

\section{Introduction}

The primary purpose of the Department of Energy (DOE), Office of Nuclear Energy (NE) Knowledge Management (KM) Program is to capture, share, disseminate, and ensure the ability to apply the knowledge created by the major nuclear energy Research and Development (R\&D) programs. In support of the KM program, the Implementation Plan for the Office of NE KM Program outlines the knowledge management and distributed data environment that is required for its success. In addition to enumerating some strategic goals and objectives, this document characterizes the initial program and identifies computer-based areas of investment required for increased knowledge sharing and collaboration. It identifies and addresses investments already in existence and describes how these investments can be further enhanced and implemented to support a distributed KM program. The Idaho National Laboratory (INL) is leading the effort to identify and address these investments through the implementation of a distributed KM program that includes participants from ten of the major DOE national laboratories.

\subsection{DOE NE Roadmap}

In the 2010 DOE NE Roadmap (DOE 2010), the DOE NE established its principal mission as advancing nuclear power as a resource capable of making major contributions in meeting U.S. energy supply, environmental, and energy security needs. To ensure that nuclear energy remains viable, DOE NE launched an R\&D program organized around four research objectives related to developing technologies and solutions to:

- Improve the reliability, sustain the safety, and extend the life of current reactors

- Enable new builds and make new reactors more affordable so that nuclear energy can help meet national energy security and climate change goals

- Enable sustainable nuclear fuel cycles

- Understand and minimize the risks of nuclear proliferation and terrorism (DOE 2011).

Research Objective 1 is primarily the Light Water Reactor Sustainability (LWRS) program. There are currently 104 nuclear power plants operating in the United States; this fleet has consistently maintained outstanding levels of nuclear safety, reliability, and operating performance (with an average capability factor above $90 \%$ ). Each reactor currently has a 60 -year operating license, and efforts are underway to extend licenses another 20 years. Without assurances that these plants can operate safely, however, the Nuclear Regulatory Commission will not grant these extensions. The LWRS program focuses on the long-term vision and support of national laboratories and universities, which address the strategic reliability and safety requirements of existing nuclear power plants that cannot be addressed by more inherently tactical organizations.

Research Objective 2, Enable New Builds and Improve the Affordability of Nuclear Power, is primarily focused on Advanced Reactor Concepts (ARC). ARC enables clean, secure nuclear energy generation through the development and demonstration of advanced thermal and fast spectrum reactor designs with significantly enhanced capabilities and performance characteristics. This will involve long-term revolutionary improvement of reactor system performance through the science- 
based development and integration of advanced materials, coolants, fuels, energy conversion, and other nuclear plant technologies.

Research Objective 3, Developing Sustainable Fuel Cycle Options, works to improve uranium resource availability and utilization, minimize waste generation, and provide adequate capability and capacity to manage all wastes produced by the fuel cycle. The key challenge is to develop a suite of options that will enable future decision-makers to make informed choices about how best to manage the used fuel from reactors. The overall goal is to have demonstrated the technology necessary to allow commercial deployment of solutions for the sustained management of used nuclear fuel that is safe, economic, secure, and widely accepted by the American people.

Each of these objectives serves as the knowledge basis for the NE-KM Program. Although the program has primarily been concerned with securing historical data, in 2011 it broadened its focus to become an integrated effort that includes information generated by the R\&D objectives.

\subsection{Business Drivers}

The major business drivers behind DOE Office of NE's increased interest in and application of KM lie in the following areas:

- Working Nationally. Office of NE's programs are spread across the major DOE laboratories, necessitating the sharing of information to effectively meet program objectives.

- Budget Constraints. With increasing budget cuts, nuclear energy programs are required to do more for less; access to knowledge becomes more important to keep up with the increased pace and workload.

- Mobile Workforce. Working across the laboratories requires program participants to be more mobile, which creates problems of knowledge continuity for R\&D activities.

- Technological Advances. Advances in information technology not only have made connectivity easier, but the expectations for sharing information have radically changed.

Given the distributed nature of the NE programs, participants have much less time to digest and analyze incoming data and information, let alone retrieve, access, and apply relevant, yet necessary, information to their programs. DOE NE recognizes the strategic importance of implementing a KM program that aligns with the business needs of the nuclear energy community. Moreover, the business case for establishing the KM Program is simple. Without an effort to immediately preserve, evaluate and disseminate existing information, it will be irretrievably lost, the money invested to create it will have been wasted and future generations will have to repeat the research if nuclear energy is to be a factor in our energy future. The Office of NE KM Program will allow the Office of $\mathrm{NE}$ to leverage knowledge for a sustainable advantage for its nuclear energy programs.

\subsection{Mission and Vision}

The Office of NE KM Program has a threefold mission to capture and make available nuclear energy knowledge to researchers and engineers, provide innovative KM technologies for easy information retrieval and collaboration, and foster continued nuclear energy education to graduate and undergraduate students throughout the nuclear energy community. The DOE NE will continue its leadership in providing both proven and innovative technologies to help meet the diverse needs of the nuclear energy program today. The department recognizes and addresses the challenges 
inherent in those needs and effectively manages the associated resources. In support of the NE KM mission, the following statement was developed:

"The Office of NE KM Program is the deliberate and systematic coordination of nuclear energy information, structured in a manner that can add value to the nuclear energy programs through reuse and innovation. This coordination is achieved through creating, sharing, and applying nuclear energy $R \& D$ knowledge as well as through teaching the valuable lessons learned and best practices to early career engineers in order to foster continued nuclear energy learning."

In the long term, the KM Program will

1. Establish a virtual knowledge center to manage, (e.g. identify, describe, format, integrate, collect, protect and disseminate) all forms of data to enable analysis relevant to the overall goals of the NE programs.

2. Lead the national and international efforts to adopt, develop, and maintain the standardization of critical data and metadata formats where users and creators are responsible to validate and improve data accuracy, consistency, and accessibility among the partners.

3. Facilitate the sharing of knowledge, providing people with easy access to and use of tools, processes, venues, and facilities to help them solve problems and achieve understanding leading to a culture where sharing knowledge is a daily activity.

4. Implement a collaborative architecture for sharing the existing knowledge that is distributed throughout the DOE NE and other partners through the adoption and support of open protocols, tools, services, and distributed data resources.

5. Protect, identify and resolve access control issues including those arising from export controlled and proprietary information.

In the short term, the program will concentrate on areas that DOE NE deems highest priority including:

1. Establish Communities of Practice responsible for developing standards and protocols for knowledge collections.

2. Identify the most important knowledge to collect within the primary programs of Used Fuel Disposition, Fuels, and Separations.

3. Within each program, develop a taxonomy for the scientific classification of nuclear energy data; organizing the information according to supertype-subtype relationships.

4. Develop and implement tools for sharing information and enhancing collaboration, e.g., face book, SharePoint, blogs, etc.

\subsection{Critical Success Factors}

As in all programs or projects, several key factors will have a significant influence on the realization of the desired outcomes. For the Office of NE KM, the five critical success factors are:

- Involvement of DOE NE stakeholders in defining program priorities, establishing the requirements, defining expected capabilities, and reviewing preliminary results to test the validity and credibility of those results;

- DOE NE leadership commitment and support at all levels, including financial support at the 
level required for program success;

- Availability of key experts at each laboratory with a commitment to encouraging a culture of sharing knowledge and supporting communities of practice;

- Continuous communication between the Office of NE program manager, the NE KM project manager, and NE stakeholders;

- A clear statement and agreement as to project objectives and desired outcomes.

\subsection{Scope}

In this section, the NE KM Implementation Plan provided a brief description of the NE KM Program, its mission and vision, a description of the Office of NE R\&D programs, the business drivers that led to the development of the NE KM program. From this point forward this plan describes the activities required for short term priorities, as defined by DOE NE, and is broken into several sections:

- Section 2 - describes the Governance that will be applied to the NE KM Program, including governance for managing the program, Communities of Practice (CoP), and the standards and procedures that will be developed to support the program.

- Section 3 - gives an overview of the knowledge collections, including both historical and knowledge being generated under today's programs.

- Section 4 - describes the collaborative technologies that will be used for the NE KM program.

- Section 5 - provides the path forward for establishing the collaborative tools.

- Section 6 - describes the key indicators that will be monitored to analyze use and performance.

\section{Governance}

KM governance is the processes and principles that act as a framework for examination, regulation, supervision, and revision of NE-KM strategies. An appropriate governance framework ensures the delivery of expected KM processes and services. Governance guides both the initial implementation and ongoing control and authority over KM strategies. It provides management of risk, review mechanisms and fiscal accountability in leveraging tacit knowledge, and sharing explicit knowledge (Zyngier, 2005). Two primary components of KM governance include the establishment of Communities of Practice (CoP) and implementation of standards and procedures.

\subsection{Communities of Practice}

Part of the strategy for the NE-KM Program involves the creation of CoP. These are networks of individuals with a common, shared purpose grouped together to facilitate knowledge building, idea creation and information exchange. CoPs will emerge from voluntary, informal workgroups formulated around a specific area of expertise integral to the NE Programs.

\subsection{Standards and Procedures}

The KM standards and procedures will provide users with direction and control that reflect the requirements of the NE program. Standards will be appropriately quantified, qualified, expressed, and coupled with KM processes for deployment and enforcement of compliance. Standards and procedures for knowledge management will include the following: 
- Standards for knowledge collection

- Knowledge definitions

- Knowledge categories and attributes

- Knowledge taxonomies and ontologies

- Metadata definitions and requirements

- Guiding principles for releasing information in a public facing environments

- Collaborative communications in a public facing environment

- Training requirements

- Quality Assurance (QA)

- Cyber Security

\section{Knowledge Collections}

Knowledge collections, regardless of whether the information is historical or current, will include both structured information and unstructured information. Structured information is information that fits well into structures such as databases, document management systems, Enterprise Resource Planning (ERP), etc. Unstructured information is the type of information that does not fit well within a structured environment such as audio files, videos, and other complex assets.

\subsection{Preserving the Past}

To date and as examples, the EBR-II, Fast Flux Test Facility (FFTF), LOFT, and Used Fuel Disposition (UFD) knowledge collections have been identified as information that is valuable to the DOE NE programs. Appendix A and Appendix B respectively provide a description of these collections. Four phases for collecting this information is required.

\section{Phase I: Identifying}

- Establish working relationship with representatives from each of the DOE laboratories; perform an information audit, e.g., identify core knowledge within each subject area.

- Identify and locate the knowledge repositories across the M\&O contractors, identify the location and structure of the knowledge.

- Identify knowledge that is in jeopardy of being lost (discarded or destroyed) and take necessary short-term preservation actions.

- Identify subject matter experts (SMEs); work with experts to prioritize the knowledge based on program needs.

- Clearly define the end users of the data, determine how the data will be used and by whom, i.e., further establish the importance of the knowledge.

\section{Phase II: Gathering and Organizing}

- Assess data characteristics, determine knowledge gaps for defined uses; add additional data sources where necessary.

- Assess the knowledge in detail, divide into logical categories, construct a structural knowledge 
taxonomy based on categories, and create electronic indexes using a predefined metadata format.

- Where necessary, seek to identify information that may not be included in existing databases and upload to electronic format; divide the information per the logical categories and within the knowledge taxonomy.

- Conduct interview sessions with subject matter experts with the ultimate goal of better understanding the captured information; employ these experts to enrich the metadata previously created.

\section{Phase III: Assess Knowledge Quality}

- Employ V\&V techniques to ensure the right information was captured and that it was captured correctly with respect to the documents, experimental data, and simulation results.

- Ensure the consistency of the previously created indexes to ensure they accurately represent the uploaded information.

- Employ data-quality management techniques for long-term management of data quality, data standardization, data duplication and maintenance of cross-reference information.

\section{Phase IV: Sharing}

- Enhance and modify collaborative tools where necessary to include appropriate protocols, tools, services, and distributed resources.

- Establish access control standards, the transfer of these documents across an unsecure internet, and the protection of the information once it is out of the control of the repositories.

- Make knowledge available via KM infrastructure.

\subsection{Capturing Progress}

The Capturing Progress activity follows the same tasks as those described in Preserving the Past, however, extends the knowledge management activities to include appropriate analysis of new knowledge resources. This is particularly important to ensure that we are consistently capturing the information that is required for current program success.

\section{Phase V: New Knowledge}

- Adaptive knowledge infrastructure is in place for new knowledge resources.

- Define standards that accurately describe the knowledge captured.

- Ensure captured knowledge gets to the right person to make decisions in a timely manner.

- Embed knowledge into a process to improve quality and consistency.

- Establish intelligence tools for capturing through archiving.

- Develop cohesive knowledge requirements for both subject matter experts and stakeholders.

- Capture knowledge from anywhere, e.g., annual off-site meetings with breakout sessions, hand-held devices, etc.

\section{Phase VI: Integrating Distributed Knowledge}


- Knowledge tools include semi-automatic creation of metadata.

- KM principals are part of Lab culture and supported by layered and expandable collaborative tools.

- Increase remote data capture and management.

- Incorporate data mining techniques where appropriate.

\section{Collaborative Technologies}

This section describes the solution concept for collaborative technologies. In most cases, these technologies are already in use at the INL and can be leveraged for the NE KM Program. To that end, specific vendor technologies are listed. In general, users will have access to wikis, blogs, discussion groups, CoP websites, document repositories, newsletters and alerts.

\subsection{Architecture}

- Reuse: Existing investments in information technologies throughout the DOE laboratory constellation shall be leveraged.

- COTS: Wherein possible commercial off-the-shelf (COTS) technologies shall be preferred over custom written applications.

- Open Standards: Data and documents generated and archived by the system shall use open standards to preserve document life-cycle viability.

- Metadata: Electronic media (files, data, documents, video, etc) shall have a mandatory workflow associated with the upload process enforcing the tagging of metadata related to the document. This metadata shall support both search and security.

- Search: The system shall utilize a federated search capability to mesh with site external content (open access / open source) as well as internal content that respects role-based access (RBAC) permissions.

- Private/Public Access:

- Private: The system shall include private collaborative workspace for internal DOE "working documents" or "working teams" whose interim products are not intended for public distribution. This system shall be characterized as an extra-net and protected via invited user credentials and secure socket layer (SSL) encryption.

- Public: The system shall include public acing access tools for dissemination of public documents as well as methods for the public to interact with DOE community representatives (e.g., public discussion forums, survey, etc.)

- User Experience: Users shall interact with the system using browser-based tools. Mobile access will be possible (though user interface will not be as rich). Generally thin-client (i.e. web browser) utilization will be sufficient, although some thick-client (e.g. Office applications (e.g. Open Office, Microsoft Office, etc.) may make a more compelling interface. The system shall be Americans with Disabilities Act (ADA) Section 508 compliant.

\subsection{Infrastructure}

- Collaboration: The system shall include the ability for extra-net users to upload documents, 
videos, data, etc.

- Storage: The system shall be capable of large scale (terabyte) file uploads (e.g., supporting the curation of CFD models and data).

- Processing: The system shall be capable of interfacing with high performance computing (HPC) for models and simulations requiring HPC capabilities.

- Bandwidth: The system shall have state-of-the-art inter-system bandwidth (model runner to HPC processor [e.g., ten gigabit connections]), as well as appropriate bandwidth for end-user file and data upload and download requirements (multiple gigabit support).

\subsection{Interface Tools}

The tools referenced below are available for immediate prototyping. Use as a DOE federated corporate tool will require licensing analysis.

- Collaboration: Microsoft SharePoint 2010: Meeting workspace, document creation workspace, process work-flow, simple team/process management, hub portal with external mash-ups.

- Project Management: Microsoft Project 2010

- Team Management: Microsoft Team Foundation Server 2010: Tasking, team-specific workflows, and customization. Ability to support the spectrum from ad hoc projects to more formalized Agile or Scrum project to very formal CMMI project management. This is leveraged best as the tasking mechanism of Project Management.

- Online Meeting: Citrix's GoToMeeting

- Online Chat/SMS Texting: Microsoft Lync Server

- Video Server: Content hosted within SharePoint so content owner can own the metadata and delivery (instead of YouTube).

- Curated Modeling Environment: Generalized Energy Modeling System (GEMS), which leverages Phoenix Integration's Model Library and Model Center Server-web-based ability to run linked, complex, high CPU required models - hooked into the INL's HPC environment delivered through the SharePoint portal.

- Automated Data Load and Parse: INL developed data V\&V system supporting the NGNP's Data Management and Analysis (NDMAS) project. Drag and drop interface exists.

- OASIES: Optimized Analysis for Strategic Integrated Energy Systems (OASIES) for the public to run energy and environmental simulations with a drag and drop, point and clock interface. Initial model is the 2050 Simulation showing trade-offs on energy and economic choices that will affect the US by the year 2050 .

- Mash-ups with Web 2.0 Technologies: DOE has limited ability to control the entire message through free media (e.g., Facebook, YouTube, Twitter). Therefore, DOE's official presence on these media will push users towards DOE hosted content while leveraging the public free content and still respecting that technology's limitations.

\section{- Communications:}

- Synchronous Communications in near-real time (e.g., face-to-face) includes GoToMeetings with scheduled events, Chat (access via web or mobile technology such as 
text messaging), and Twitter.

- Asynchronous Communications are communications that include discussion boards and threads, recordings of GoToMeeting events, videos (hosted on DOE servers which eliminates the limitation requirements of YouTube), and robust and federated searches using Needle.

\subsection{Security}

- Continuous Surveillance, for all collaborations, will be monitored automatically against key word/phrase/patterns. All content uploads will be scanned and embargoed if fail. Administrators will have the ability to generate reports of activity and content (Trend Micro's Portal Protect).

- Encryption will be required for transmission of non-public content (SSL 128 bit encryption).

- Enclave; the extranet portion on the site shall be managed as a Moderate Enclave.

- Credentials: Users with logon credentials shall be managed according to the standard outlined in the Cyber Security Master Plan for the Office of Nuclear Energy Knowledge Management Program.

- Dynamic Vulnerability Analysis will be done on all custom codes/components; codes and components shall be inspected for vulnerabilities utilizing automated programming dynamic analysis prior to employment on the site (HP's Fortify).

- Static Vulnerability Analysis will be performed on all hosted content and subjected to continuous analysis on the site (NeXpose Rapid7).

\section{Scheme of Maneuver}

Following are the steps that can be immediately taken to provide DOE NE with the tools needed to enhance collaboration across the DOE NE complex and DOE laboratories. These tools will enable sharing of essential knowledge to complete program tasks.

\subsection{Collaboration Portal - Extranet}

Establish an "extranet" portal for inter-laboratory and DOE - laboratory collaboration. This internal collaboration portal will immediately enable the following listed capabilities to be used as a basis and foundation to add additional capabilities as the community is prepared to receive them. The below-listed capabilities are available immediately (can be made available outside the INL firewall within one business week). The following licensing caveats and budgeting constraints apply.

\subsubsection{Capabilities}

1. Centralized management and governance of the central portal hub will be required. In other words, this (DOE sponsor org DOE-NE) program office will tightly govern and control the central and/or mature portions of the collaboration portal. This segment will be available to all system users.

2. Decentralized enablement is an increasingly liberal governance and control policies for new/immature or adhoc segments. The concept is to create enablement in preference to control. This will allow teams to determine and define the ways and means of their 
collaborative interaction. Access control to these segments will be limited to segment participants.

3. Distributed access management allows community managers to control membership and activities of their segments (i.e., education of information security and monitoring/control community behavior for their users will be reserved at this level).

4. Local design allows community managers and their "designers" to build-out their segment's capabilities appropriate to the community's need for collaboration and sharing of information.

5. Role-based access (RBAC) gives users access to their self-created information as well as across all the various segments based upon their access rights granted by community managers.

6. Document capabilities provide users with the ability to upload documents, metadata assignment, and search-ability. The system shall have the ability to enable users to upload documents/data according to rules to-be-determined (TBD). Those TBD rules shall be capable of grading appropriate to the needs of the local community's mission (i.e., curated files may need $V \& V$, version control and document controls, but others may not.

7. Records management will be incorporated and provide users with the capability of workflow and records keeping required under DOE and Public Records requirements, and shall be able to be designated as a "Satellite Records Management System" in accordance with DOE rules and regulations.

8. Change notification automation allows users of the system to change their method of notification of information change for each community/functional segment. Users shall be able to control notification based upon user preference, i.e., notification of minor or major changes, notification of change content, delivery immediately or as a daily/weekly digest.

9. Discussion Groups: The system shall enable community managers to create threaded discussion groups at will. Users will be able to subscribe to feeds. Communication shall be either web or email/mobile enabled (or both).

10. Online Collaborative Document Authoring: The system shall be capable of enabling online synchronous document collaborative authoring in near-real time. The system shall enable online asynchronous document authoring with version control.

11. Offline Capability: The system shall enable off-line synchronization of content to enable use of content and change of content when users are not network connected.

12. Calendaring: The system shall enable full calendaring capability at each appropriate community segment. The system shall also include the ability to combine any or all calendars according to RBAC rules.

13. Task Management: The system shall enable community managers to engage with community members with embedded task management. The system shall enable robust task management that can be modified according to the tasking artifacts and rules associated with professionally managed projects ranging from project management, software management, or anything approaching or exceeding the rigor of CMMI (Capability 
Maturity Model Integration) or PMI (Project Management Institute). The purpose is to ensure the ability to create and manage tasking assignments associated with Nuclear Quality Assurance 1 (NQA-1) level of rigor.

14. Workflow: The system shall be capable of simple (three-state, approval process) and complex workflows. Workflows shall be re-useable (to leverage development efforts associated with building complex workflows).

15. Accessibility: The system shall be Section 508 compliant. The system shall be functional (although degraded functionality is acceptable) using mobile devices (tablets/smartphones).

16. Security. The system shall be scanned for inappropriate content/embargoed content upon upload. Administrators shall be able to run reports and search for emergent identified problems.

17. Foreign Access. Using RBAC create a method which enables foreign science/engineering collaborators to interact with domestic partners and laboratories.

\subsubsection{Caveats}

1. Licensing is required for an instance outside the firewall. Cost is approximately $\$ 40 \mathrm{~K}$.

2. Collaborative document authoring requires that all users have a licensed version of Microsoft Office 2007 or later. If not, a client access license (CAL) must be purchased at \$130.00 per non-conforming user.

3. Complex workflow creation without an IT practitioner will require an appropriate COTS package like $\mathrm{K} 2$ at the cost of $\$ 50 \mathrm{~K}$ per enterprise servers. This enables workflow owners or subject matter experts to establish and maintain their own workflows.

4. Federated search (across multiple systems) will require an Enterprise license of SharePoint (\$8K per web-front-end). Simple search (within the single server instance) will function at no additional license charge.

5. Management and administration of this portal is characterized as a level-of-effort activity. As soon as multiple users are added and the ability to grow the user base according to community segment needs, the requirement for user support, management and administration will be triggered. At a minimum the following items must be considered:

a. Management. A manager must be designated as a single point-of-content for change management, future-proofing, and utilization decisions or the portal.

b. Administration. A system administration (sysad) team must be designated who can respond to requests for new user accounts and assignments, system utilization (load balance, data management) and technical requests for system integration activities with external servers/capabilities.

c. End user support. A help-desk function must be staffed to provide a personal point-of-contact for assisting inevitable "how do I get access to...?" to "how do I do [x]...? calls. A documentation effort must be made taking into account the needs of adult learners to integrate and embed appropriate just-in-time (JIT) learning aids 
(i.e., online help, standards based interface, embedded section 508 compliance formatting/content issues management).

6. Staffing - at a minimum permanent staffing for such an endeavor will start at two (a system manager and a technical system administrator) and must expand as needed to comply with growth, usability, and acceptable customer satisfaction levels.

\subsection{Collaborative Portal - Internet}

Establish an "internet" portal for DOE-NE public outreach. External public facing portals are generally used as marketing or static-information hosting portals, although some interactive capability can be appropriate (limited to an analysis of bandwidth and CPU requirements). Generally, the interaction components are things like surveys or hosting of public-outreach oriented models, simulations or videos. This portal will immediately enable the following listed capabilities and will be used as a basis and foundation to add additional capabilities as the community is prepared to receive them. The below-listed capabilities are available immediately (can be made available on the INL DMZ within one business week) within the following licensing caveats and budget constraints.

1. Federated search capabilities found in Needle, including the new content approved for public release within the Internet and extra-net portals as well as federated search with other SOA compliant DOE search results provides.

2. Knowledge Encapsulation Framework (KEF) developed at PNNL to serve as an interface into restricted (AT) data from FFTF design, construction and operation and provides a standalone KM environment for data entry that will be adapted to provide a searchable interface for integration with Needle.

3. Online surveys that provide an ability to gauge visitor acceptance and needs, or to ascertain public response to current issues.

4. Online modeling, hosting interactive models and simulations

5. Online video, which hosts a library of DOE and Laboratory videos displayed according to the DOE NE needs. Funnel Facebook and YouTube users to the DOE presentation of this material making this material searchable.

\subsection{Project Management Tools}

Team management tools (i.e., task management and workflow) are available immediately. Project management requires acquisition of a project management server (e.g., Microsoft Project) coupled to a project performer capability (e.g., Microsoft Team Foundation Server) all served in a webbased environment. There is a heretofore much needed capability between planners (managers) and doers (knowledge workers) that is enabled through web-based project interaction. This bridges the gap between planning and execution and enables the automated collection of project metrics, which reduces response workload back to managers on the behalf of performers.

\section{Key Performance Indicators}

Key performance indicators will be regularly measured to determine the effectiveness and usability of the collaboration suite of tools. Key performance indicators include the following:

- Time from data acquisition to final product delivery

- Site usage, i.e., number of unique pages viewed 
- Sites created, i.e., number of websites, wikis, blogs, etc., created by users

- Number of content downloads

- User satisfaction -- satisfaction scores based on semi-annual survey results

\section{Summary}

In summary, the basic technology components required to establish the Office of NE KM Program can be immediately implemented. In most cases, these technologies already exist at the INL and implementation then becomes the simple task of prioritizing which are most important according to KM funding. Immediate steps can be taken to preserve the past and ensure the collection of all knowledge being created now. As more is learned about the explicit and tacit knowledge that is required by the NE programs and as funding increases, the system can be enhanced to include all technologies described in this plan. It will be important to develop a schedule for collecting tacit knowledge, as this will be a top priority over the next two years. 


\section{References}

[1] S. Zyngier. Knowledge Management Governance, Article for the Encyclopaedia of Knowledge Management, Ed. David Schwartz, Idea Publications, Hershey, 2005.

[2] PC Ellis, R.S. Butner, Interfacing Knowledge Encapsulation Framework with NEEDLE/HAYSTACK, Version 1.1, September 2010, Pacific Northwest Laboratory.

[3] Energy and Water Development Appropriations Bill, 2012, House of Representatives, $112^{\text {th }}$ Congress $1^{\text {st }}$ Session.

[4] Final Report Total System Performance Assessment Peer Review Panel, February 1999. 


\section{APPENDIX A}

\section{FFTF Knowledge Collections}

\section{Introduction}

There has been little funding to continue the FFTF information collection and assessment effort; the information in these documents will be invaluable for research and development as a prelude to a new advanced LMR, such as an advanced Burner Reactor (ABR). The information will also be invaluable for benchmarking advanced computer simulation models against realistic reactor operating and performance characteristics. The modeling and simulation effort is a key effort within the Fuel Cycle Technology (FCT) program and could be a mainstay effort in future years. With decommissioning of the FFTF reactor imminent, it becomes important to retrieve and securely store this information for future use within the FCT program. There are four general categories of FFTF related information:

- Literature related to the design, development and operation of FFTF comprised of 3000-6000 documents, most in journal publications, ANS meeting summaries, technical conference reports, technical reports published by the Hanford Engineering Development Laboratory (HEDL), Westinghouse Advanced Reactor Division (ARD), as well as some unpublished reports. A bibliography of these reports needs to be produced, and the documents or references need to be made available.

- Over 500 boxes of FFTF related data need to be made available electronically. A catalogue of this data exists as a cryptic document that needs to be expounded and converted to electronic form, including a FFTF Data "wiki" that describes the metadata for each data set.

- Coupons are material samples that were exposed to known fast fluences within FFTF and exist as physical samples stored in hot cells at Hanford. The data related to these coupons, which cost hundreds of millions of dollars to produce, need to be catalogued and made available electronically.

- The drawing sets of the FFTF, including its major structures, systems, and components (SSC) need to be catalogued and made available electronically.

\section{FFTF Information for Research and Development}

The FFTF records consist of basic design information such as codes and standards, system design descriptions, operations manuals and procedures, safety analysis reports and safety evaluation reports, and system-acceptance test reports. In addition, the records contain the approaches and procedures by which the basic performance of the reactor plant was designed and verified.

As FFTF is currently in the process of being decommissioned, there was a growing time-critical danger that these records could be discarded or archived with insufficient cataloguing to be of value to the FCT. This program, during Phase I of its effort, reviewed, categorized, and securely stored these documents for future use. In addition, several of the document storage systems containing FFTF information were exercised to validate their retrieval characteristics.

\section{FFTF Information for Modeling and Simulation}

Examples of test cases that can be constructed for modeling and simulation from FFTF information include, but are not limited to, the following: 
- Irradiation swelling and creep produce bowing of the assemblies in a fast reactor core that eliminates the normal space between adjunct assemblies and has the potential to lock the assemblies into the core. To prevent this, it is common practice to rotate or rearrange the assemblies at each refueling outage. Measurements of the load required to remove the assembly from the core are available and can be used to provide test case for modeling and simulation codes calculating core performance.

- When a sodium cooled fast reactor is taken up to full power, a significant amount of thermal distortion occurs in the primary and secondary piping systems. Measurements of this thermal movement are available for use in providing test cases for modeling and simulation codes. When properly tested against these cases, the codes can be used to reconfigure the primary and secondary systems to minimize the space required and thereby reduce the cost of any reactor plant under design.

- Just prior to the shutdown of the FFTF in 1992, a series of passive safety tests were performed. In these tests, a loss of flow without scram was performed from $50 \%$ of full power. This event demonstrated that a LMR can under such an event and that the flow will coast down to that rate established by natural circulation without excessive fuel or coolant temperatures. Subsequent to this, passive safety and the ability to undergo such an event became sine qua non of all modern LMR design approaches. The experimental data and other records from this testing are being retrieved for use as test cases for modern design computer simulation codes and models.

In addition to these examples, many other test cases including virtual design of the reactor plant based upon actual design and performance information and data can be developed.

\section{Current Status of FFTF Information Collection Program}

Archiving of FFTF information, documentation, and data began in 2009. A plan was developed and actual physical and categorization of documentation was conducted. Approximately 100 boxes were categorized consistent with the IAEA LMR taxonomy and are being held in a secure storage location at PNNL. A proposed budget to continue the activity through next fiscal year, including responding to requests from the Modeling and Simulation campaign, responding to requests from the Industrial Consortia, and likewise converting documentation to electronic format for inclusion in an electronic database has been developed and is included herein. Examples of the kinds of documents that exist are summarized below:

- Preliminary Safety Analysis Report, Final Safety Analysis Report, and supporting safety analysis documentation.

- Technical Specifications (Tech Specs) defining the technical parameters and operating limits for the reactor plant.

- System Design Descriptions (SDD).

- The Basis for the Tech Specs which provide the technical rationale for the operating parameters.

- The Authorization Basis defining the approved conditions for operating the reactor plant.

- Control Room operating procedures for the reactor plant.

- Reactor Development and Technology (RDT) Standards which define the design and procurement specifications for all major structures, systems, and components.

- Hanford Engineering Development Laboratory (HEDL) Standards that identify the additional conditions and waivers applied to the RDT Standards. 
- Component procurement specifications that define the precise technical provisions applied to the procurement of all major components in the reactor plant.

- Reload Design Reports (RDR) that define and specify the conditions and limits under which each core reload was to operate.

- Unusual Occurrence Reports (UOR) which identify and characterize any anomalies or unanticipated conditions that occurred during operation of the reactor plant.

- Control Room Log Books which document actual actions taken during operation at power as well as during refueling and maintenance outages.

- Detailed Refueling Plan (DRP) that documents how experiments were handled and managed after they arrived at the reactor plant and how driver fuel assemblies were managed within the reactor plant.

\section{M0-276 Library}

The fuel test results information at the M0-276 Library exists in several different formats depending upon the final stage of the test evaluation. In some cases, there is information from both non-destructive and destructive examination while in other cases only non-destructive results are available. Non-destructive information includes disassembly records, dimensional profilometry, gamma spectrometry, and neutron radiography. Information from destructive examinations includes fission gas analysis, metallographic and associated photomicrographs. The Library also contains the background literature used in the design of the tests. Finally, it contains information on many precursor tests performed in EBR-II. Examples of the kinds of information available in the Fuels Testing Library are discussed in Butner, Wootan, Omberg, and Makenas [1] and are:

- Design and results information from the Core Demonstration Experiment

- Design information on instrumented tests

- Experimental results from the Driver Fuel Evaluation Program

- HT-9 and D-9 cladding and duct irradiation swelling characteristics

- Design and results information from high burnup metal fuel tests (188 Mwd/kg)

- Design and results information from high burnup MOX fuel tests (238 Mwd/kg)

- Advanced cladding and duct procurement specifications

- Driver fuel procurement specifications

- Vendor driver fuel certification data

- Driver fuel and test design steady-state fuel performance computer codes

- Driver fuel and test design transient fuel performance codes

- Cladding density measurements

- Burnup measurements

- Microprobe scans

Information, documents, and other records from FFTF are currently being stored and have been stored for some time in the MO-276 library. All of the information in the library is in the form of hard copies or microfilm reels and needs to be put into electronic storage forms. The industrial consortia within the FCT program have expressed interest in obtaining some of the information in the library, and as this information will be retrieved for future research and development within the FCT program as needed. To facilitate this, current planning is to convert it to an electronic format as soon as possible. 


\section{Handling of FFTF Applied Technology}

At least some, and possibly much of the information being retrieved may be AT. Pending further review and AT guidance, PNNL is managing this activity using the DOE NE Guidance for managing AT documents. As this activity involves both PNNL and FFTF CH2M Hill, PNNL coordinates very closely with the Site Operations Office (RL) Lead for FFTF.

Short-term activities require a coordinated and parallel effort for information collections at each relevant site. We recommend first attempting an agreed upon proof-of-concept process where we identify a selected set of documents, run the documents through the process, and refine the process where necessary. Recommendations to the IAEA for an approved taxonomy could also be incorporated into the proof-of-concept process.

All documents collected under this program will be sent to OSTI. However, it is recommended that OSTI be viewed as a common repository and not as a knowledge management solution; it must be easy for researchers to retrieve the right information. For this to occur, need-to-know AT policies and procedures must be developed that are program based, rather than case-by-case based. Additionally, laboratories must allow the information to be stored in an approved knowledge management system. 


\section{Appendix B}

\section{Used Fuel Disposition}

\section{Nuclear Waste Disposal}

Despite the Administration's decision to close Yucca Mountain, the DOE remains under obligation to continue the congressionally-mandated activities to provide for the development of repositories for the disposal of high-level radioactive waste and spent nuclear fuel. Funding has been recommended in the Energy and Water Development Appropriation Bill, 2012 to begin research and development activities to provide alternative solutions to Yucca Mountain. Moreover, while Yucca Mountain may continue to be viewed as one of these solutions, the analysis of new solutions will require inclusion of the information that was gathered from the work completed on Yucca Mountain. That is, proposed alternatives must be able to show how and why these solutions are better than the existing option. For the analysis to be successful, it is important to identify, assess, prioritize and make accessible the YMP information. Examples of the types of information that may be required are summarized below:

- Existing law and congressional direction

- Design data and analysis

- Waste form studies with analysis results and review criteria

- Scientific merit of Yucca Mountain disposal site with comparative analyses

- Used fuel disposition, storage, transportation, and repository studies

- Modeling and simulation data to support future probable behavior of the repository with review criteria

- Repository acceptance criteria

- Quality assurance policies, procedures and records

- Inspection data

- License application activities

- Technical merit and cost-benefit analysis associated with interim storage of spent fuel and other high-level nuclear waste at permanently shut-down reactors

- Proposed long-term disposal site alternatives along with scientific merit and comparative analysis

- Cost comparisons and acceptance criteria of alternative disposal sites based on Yucca Mountain

- Utilities and their associated waste inventories, both past and future expectations

- Owners of commercial spent nuclear fuel

Initially it is reasonable to expect that the Nuclear Waste Disposal program will focus on the more urgent matter of addressing the fuel that is stored on site at decommissioned nuclear reactors. Prominent studies, e.g., The Future of Nuclear Power done at MIT, suggest that the consolidation of fuel from decommissioned sites be accelerated. DOE is looking at consolidating this high level waste based on recommendations from the Blue Ribbon Commission; the Blue Ribbon Commission is expected to make a statement about consolidation at the end of July 2011.

As the lead Laboratory for Yucca Mountain activities, Sandia National Laboratories (SNL) holds much of the information that was generated during the past several decades. Some of the 
information was originally archived in the YMP SharePoint site; however, most of the archived information was obtained from other YMP information systems operated by the M\&O contractors. Systems include (but are not limited to) RisWeb, CDIS, DIRs, TDMS, CAP, etc. Metadata for the M\&O systems was captured regularly and is made accessible through the License Support Warehouse. In support of the Nuclear Waste Disposal program, it will be important to identify all M\&O contractor systems and determine a path forward for making the highest priority information available to the program. 